\title{
Retrodiscal Approach of Lumbar Epidural Block
}

Chul Kim, M.D., Chang Jin Moon, M.D., Hee Eun Choi, M.D., Yongbum Park, M.D.

Department of Rehabilitation Medicine, Sanggye Paik Hospital, Inje University College of Medicine, Seoul 139-707, Korea

Objective To compare the technical strengths and weaknesses between retrodiscal (RD) and conventional subpedicular (SP) approaches of transforaminal epidural block (TF-EPB).

Method Sixty-one patients with L5 radiculopathy who planned to undergo TF-EPB were consecutively enrolled as study subjects. Subjects were randomly assigned to one of two groups. For the RD approach, the positioning of the patient and the C-arm were similar to that for lumbar discography. We compared the pattern of dye spreads, the frequency of complications during the procedures, and the effect of the pain block 2 weeks after the procedure between the two groups.

Results For the RD group ( $\mathrm{n}=24)$, the contrast dye diffused around the L5 and S1 nerve roots in 16 cases (67\%), but it diffused around only the L5 root in 27 cases $(73 \%)$ in the SP group $(n=37)(p<0.05)$. Two weeks after the procedure, the visual analogue scale (VAS) decreased by the same amount in both groups (RD group: 3.1 \pm 1.6 , SP group: $3.2 \pm 2.6$ ). Symptoms of nerve root irritation occurred in 1 case of the RD group and in 10 cases of the SD group $(\mathrm{p}<0.05)$.

Conclusion The RD approach was as efficient as the SP approach for temporary diagnostic relief and offered considerable advantages, such as lower nerve root irritation possible lower risk of vascular injection. Thus, it could be a useful technique when a herniated disc segment is stuck or when the foraminal stenosis is severe.

Key Words Spinal, Injection, Lumbar, Transforaminal, Retrodiscal

\section{INTRODUCTION}

Epidural steroid injections have been used for the treatment of lumbar radicular pain since 1952. ${ }^{1}$ An epidural steroid injection procedure can take an interlaminar, caudal or transforaminal approach, depending on the particular spot where a needle is inserted into the

Received June 16, 2010; Accepted January 11, 2011

Corresponding author: Yongbum Park

Department of Rehabilitation Medicine, Sanggye Paik Hospital, Inje University College of Medicine, 761-1, Sanggye 6, 7dong, Nowon-gu, Seoul 139-707, Korea

Tel: +82-2-950-1390, Fax: +82-2-935-3076, E-mail: swc328@naver.com

(c) This is an open-access article distributed under the terms of the Creative Commons Attribution Non-Commercial License (http://creativecommons.org/ licenses/by-nc/3.0) which permits unrestricted noncommercial use, distribution, and reproduction in any medium, provided the original work is properly cited.

Copyright ( 2011 by Korean Academy of Rehabilitation Medicine epidural space. The transforaminal injection approach is preferred when compared with the other two approaches because delivery of a therapeutic injectate transforaminally at the involved nerve root maximizes steroid concentration at the site of a pathology, and it can be given in to the anterior epidural space of the symptom-associated nerve root. ${ }^{2,3}$ The transforaminal approach can be divided into three sub-approachessubpedicular approach, retroneural approach and retrodiscal approach-depending on where the needle is advanced. Among them, the subpedicular approach is currently the one used most frequently in clinical practice. In this approach, when an injection needle is advanced into the safe triangle under the inferior aspect of the pedicle, as described by Bogduk ${ }^{4}$ it is positioned in the superolateral aspect of the symptom-associated 


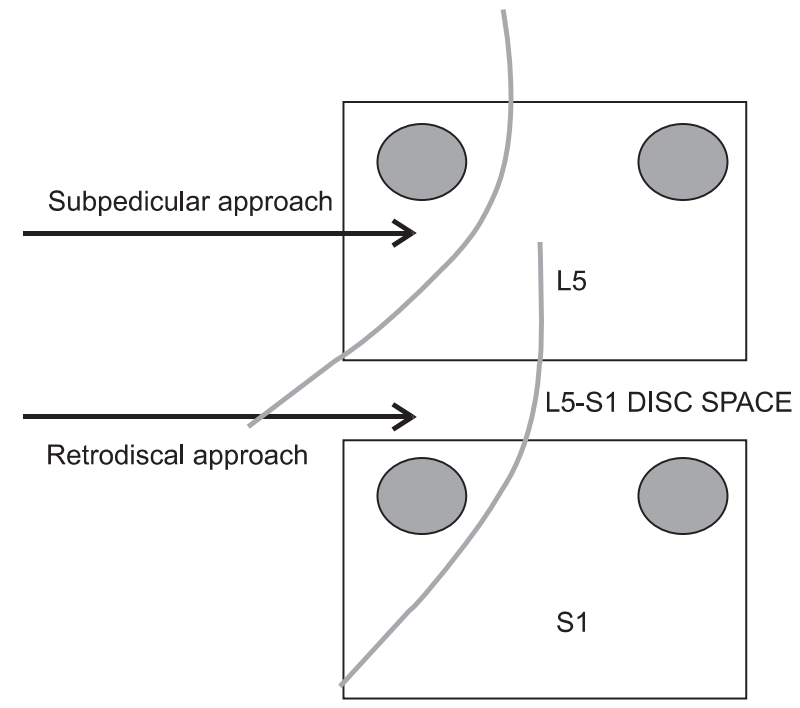

Fig. 1. Schematic description for transforaminal epidural steroid injection with the retrodiscal approach versus the subpedicular approach.

spinal cord. In this position, a drug can be injected into the anterior epidural space, or the site of inflammation between the posterior aspect of the prolapsed intervertebral disc and the anterior aspect of the dural nerve-root sleeve, and the needle passes through the superolateral edge of cervical intervertebral foramen, so there is less risk of damaging the dura mater. ${ }^{1,2}$ However, in several situations, it is difficult to place the needle through the safe triangle in the anterior epidural space, such as in cases of severe foraminal stenosis, epidural fibrosis, or progressive degenerative disc diseases. Furthermore, as the injection is given to the anterior epidural space across the intervertebral foramen, it may cause damage to the nerve root. Similarly, if the drug is injected into arterial blood vessels distributed around that space, it is likely to result in complications..$^{5-7}$ Jasper $^{8}$ proposed the retrodiscal approach as an alternative to the subpedicular approach, because the needle advanced into the inferior aspect of the foramen carried less risk of the injection entering the anterior radicular artery. This technique also had the advantage that the injection could be made closest to the nerve root, which is stimulated by a prolapsed intervertebral disc (Fig. 1). However, there has been no study to date comparing the epidural blocks by the retrodiscal approach with other possible block techniques, with regards to their differential effects and advantages/disadvantages.
Table 1. Patients Characteristics

\begin{tabular}{lccc}
\hline & RD & SP & Total \\
\hline $\begin{array}{l}\text { Number of } \\
\text { subjects }\end{array}$ & 24 & 37 & 61 \\
$\begin{array}{l}\text { Male/Female } \\
\text { Age (years) }\end{array}$ & $13 / 11$ & $11 / 26$ & $24 / 37$ \\
$\begin{array}{l}\text { Pain duration* } \\
\text { (<2 weeks/ }\end{array}$ & $14 / 10$ & $10 / 27$ & $24 / 37$ \\
$>2$ weeks) & & & \\
$\begin{array}{l}\text { Pain side } \\
\text { (Rt/Lt) }\end{array}$ & $11 / 13$ & $18 / 19$ & $29 / 32$ \\
\hline
\end{tabular}

Values are mean \pm standard deviation

RD: Retrodiscal group, SP: Subpedicular goup

*Number of subjects with pain for $<2$ weeks and $>2$ weeks

The present study attempted a comparison between the subpedicular approach and the retrodiscal approach in performing a transforaminal epidural block in patients diagnosed with L5 radiculopathy. For this purpose, we used fluoroscopy to compare the spread and location of the contrast medium and investigated temporary diagnostic pain relief and possible complications that may occur during injection.

\section{MATERIALS AND METHODS}

\section{Subjects}

The subjects of this study were selected from patients diagnosed with L5 radiculopathy upon their visit to our hospital's Department of Physical Medicine and Rehabilitation. Patients suffering from lumbago or lower extremity radiating pain were deemed to require a transforaminal epidural block because they did not respond to the conservative treatment. The final sample of subjects consisted of patients in whom the L5 nerveroot compression, by either L5-S1 herniated disc or foraminal stenosis, was observed by 3 -dimensional computerized tomography (3D CT) of the lumbosacral spine and who were diagnosed with radiculopathy on electromyography (EMG). However, we excluded patients who had received a steroid prescription or injection in the last three months from our study. We also excluded patients with a medical history of diabetes, systemic peripheral neuropathy, cerebral infarction, inflammatory joint diseases, and/or cauda equine syndrome. 


\section{Clinical characteristics of the subjects}

This subject included a total of 61 subjects who were randomly assigned to one of two groups: a "subpedicular approach" group of 37 patients (mean age $=59.4 \pm 10.1$ years) and a "retrodiscal approach" group of 24 patients (mean age $=57.2 \pm 12.1$ years). Table 1 shows the groups' male to female ratios, painful periods, and pain locations (right or left). There was no significant difference between the two groups in the characteristics of the detected lesions on the 3D-CT scans (Table 2).

\section{Randomization}

After obtaining information about their basic characteristics, the investigator randomly assigned the subjects to one of the two groups by a simple coin-toss method (simple randomization). Thus, the subjects were

Table 2. Findings of Lumbosacral 3D Computerized Tomography

\begin{tabular}{lccc}
\hline & $\begin{array}{c}\text { RD } \\
(\mathbf{n}=\mathbf{2 4})\end{array}$ & $\begin{array}{c}\text { SP } \\
(\mathbf{n}=\mathbf{3 7})\end{array}$ & p-value \\
\hline Bulging disc & 2 & 4 & 0.749 \\
Herniated disc & 15 & 24 & 0.851 \\
Sequestrated disc & 5 & 5 & 0.451 \\
Foraminal stenosis & 2 & 5 & 0.694 \\
\hline
\end{tabular}

RD: Retrodiscal group, SP: Subpedicular group assigned to the retrodiscal-approach group when they received heads and to the subpedicular-approach group when they received tails.

\section{Injection approaches}

The retrodiscal approach: Each patient was placed in the prone position with a pillow under the lower abdomen and above the iliac crest to reduce lumbar lordosis. The cephalo-caudal angle of the C-arm (KMC 950, KOMED, Kyunggi, Korea) was adjusted, allowing the incident X-ray beam to be parallel to the inferior and superior end plates of the L5-S1 intervetebral disc. Then the right and left angles of the $\mathrm{C}$-arm were rotated again towards the lesion site by $40-50$ degrees so that the S1 superior articular process was positioned and seen in the middle of the intervetebral disc (Fig. 2). At this position, a 22-gauge 5 -inch spinal needle was directed to the middle of the intervetebral disc, using the lateral aspect of the S1 superior articular process. Following that, it was inserted into the skin to be parallel to the incident X-ray beam. The needle was advanced until the needle tip reached the outer wall of the disc (i.e. the annulus fibrosus). In this process, special care was taken for the needle not to penetrate the annulus fibrosus of the intervetebral disc, with particular attention given to the anteroposterior and the lateral $\mathrm{C}$-arm images. Once the final position
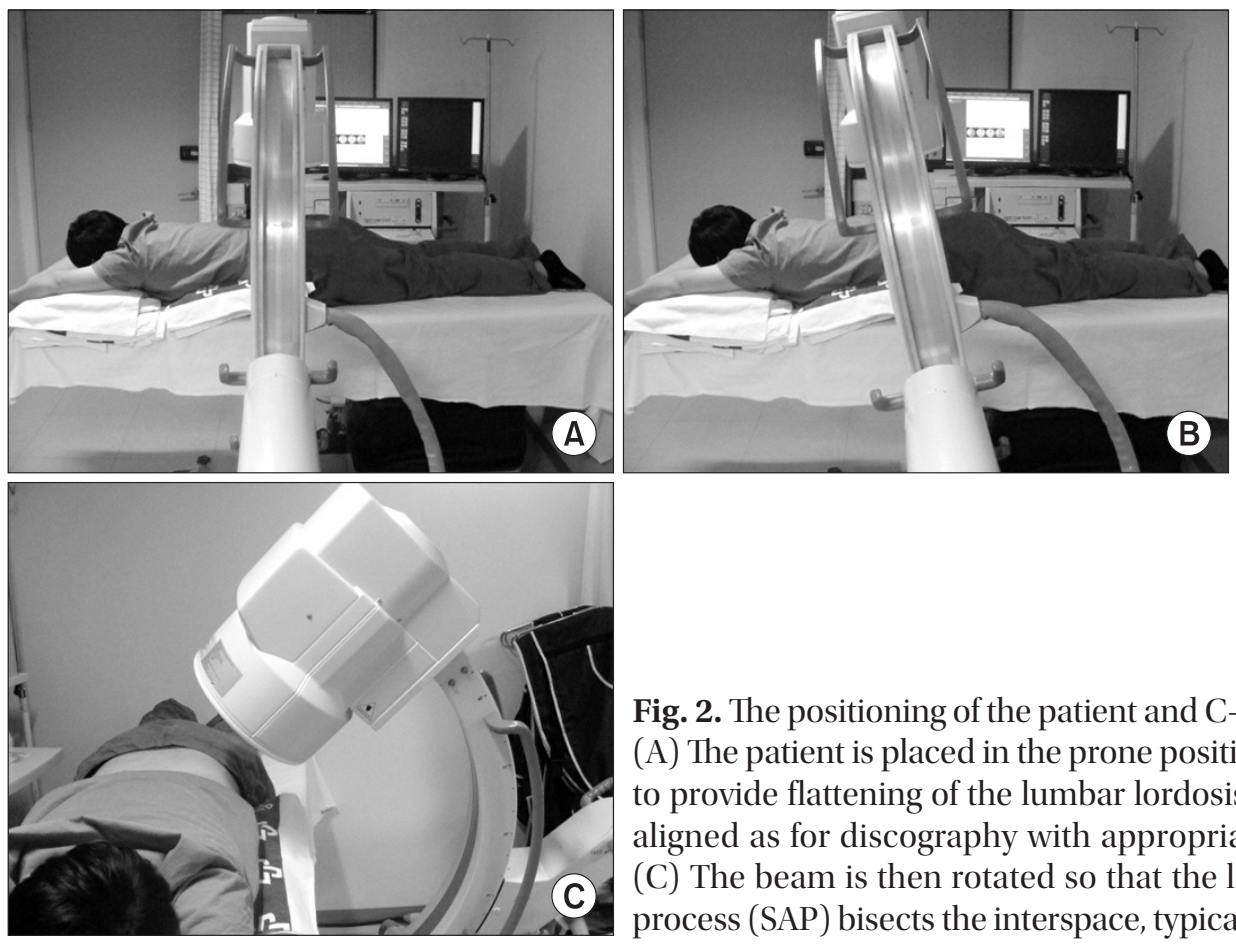

Fig. 2. The positioning of the patient and C-arm are similar to lumbar discography. (A) The patient is placed in the prone position on a fluoroscophy table top padded to provide flattening of the lumbar lordosis. (B) The targeted disc's endplates are aligned as for discography with appropriate caudal or cranial tilt of the C-arm. (C) The beam is then rotated so that the lateral surface of the superior articular process (SAP) bisects the interspace, typically 40-45 degrees off the AP axis. 
of the needle tip was determined, the investigator administered $1 \mathrm{cc}$ of nonionic contrast (Omnipaque 300; GE Healthcare, Carrigtohill, Cork, Ireland to observe the spreading location and range of the contrast agent. Then a prepared $2 \mathrm{cc}$ drug solution ( $1.5 \mathrm{ml}$ of $0.5 \%$ lidocaine + $20 \mathrm{mg}$ of triamcinolone) was injected (Fig. 3).

The subpedicular approach: In accordance with current practice, a 22G 5-inch spinal needle was inserted into the safe triangle of the lumbar spine (L5). Once the final needle-tip position was determined by the C-arm, the investigator administered $1 \mathrm{cc}$ of nonionic contrast to observe the spreading location and range of the contrast agent. Then, similar to the previous approach, the same prepared $2 \mathrm{cc}$ drug solution was injected $(1.5 \mathrm{ml}$ of $0.5 \%$ lidocaine $+20 \mathrm{mg}$ of triamcinolone) (Fig. 4).

\section{Assessment variables}

All significant events that occurred during and after the injection were recorded. The spreading locations and ranges of the contrast agent and the feelings of discomfort upon injection were observed to compare the advantages/disadvantages of the two injection approaches. In addition, to compare the two injection approaches in terms of temporary diagnostic pain relief, the patients were assessed on the visual analogue scale (VAS) before treatment and two weeks after treatment.

\section{Statistics}

The chi-square test and the independent t-test were used to compare the clinical characteristics of the two studied groups. The results of 3D-CT scans were compared using the Fisher's exact test. This test was also used for comparison of possible complications and feelings of discomfort, which could occur in the two groups during injection. The independent $t$-test was used to compare the temporary diagnostic pain relief after the injection treatment (as measured on the VAS). Data were analyzed with a statistical significance level of $p \leq 0.05$, using the SAS Enterprise Guide 4.1 (4.1.0.471) software.
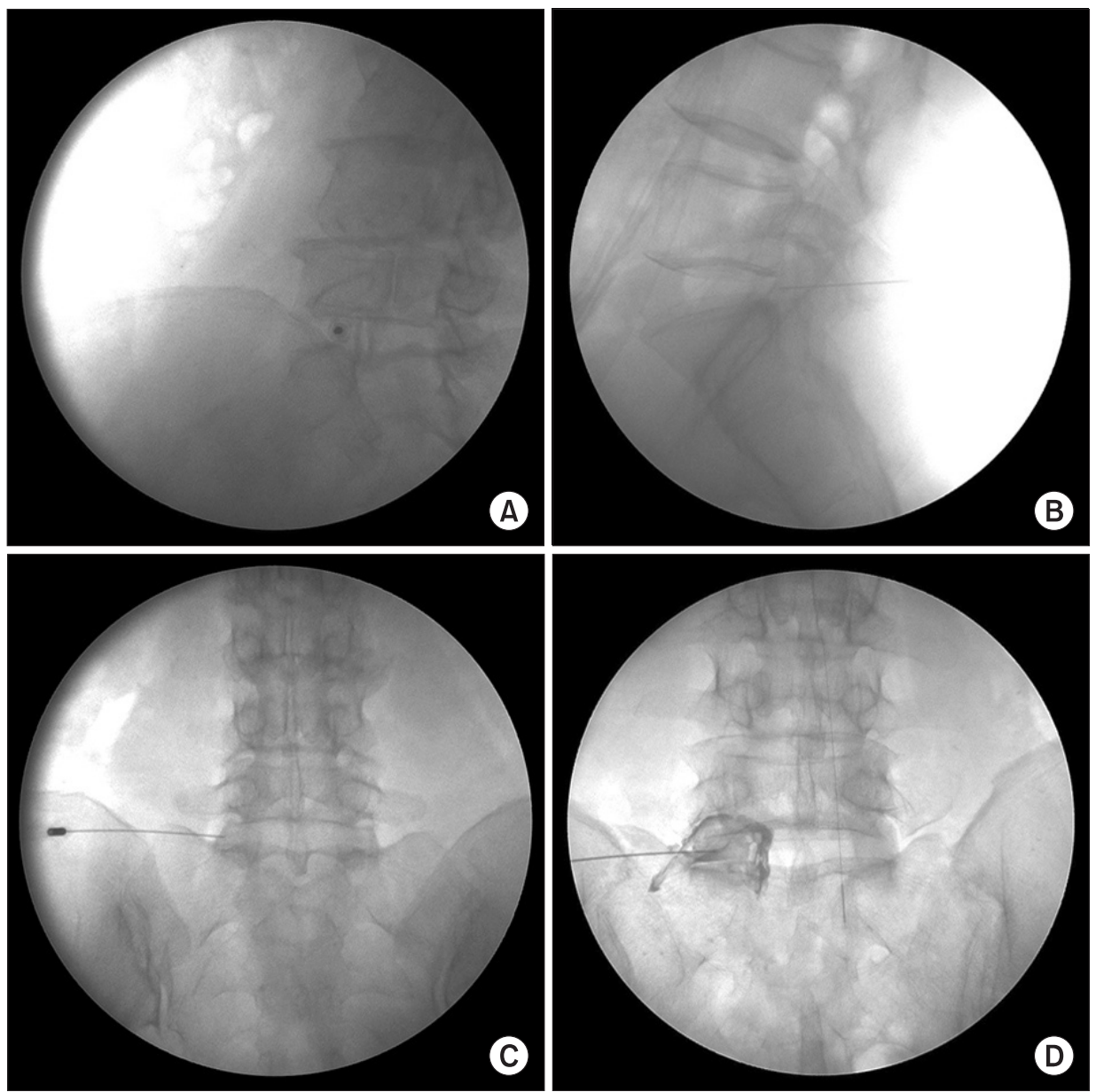

Fig. 3. Retrodiscal injection L5S1. (A) In oblique view, needle tip is advanced slowly and cautiously past the SAP lateral surface. (B) The lateral radiography should also be used while advancing past the SAP to minimize the risk of the penetration, while the resistance to the needle advancement is also used as sign to stop. (C) The AP view will most often demonstrate the tip in the interpedicular line. (D) A small amount of contrast is used to confirm epidural spread. 

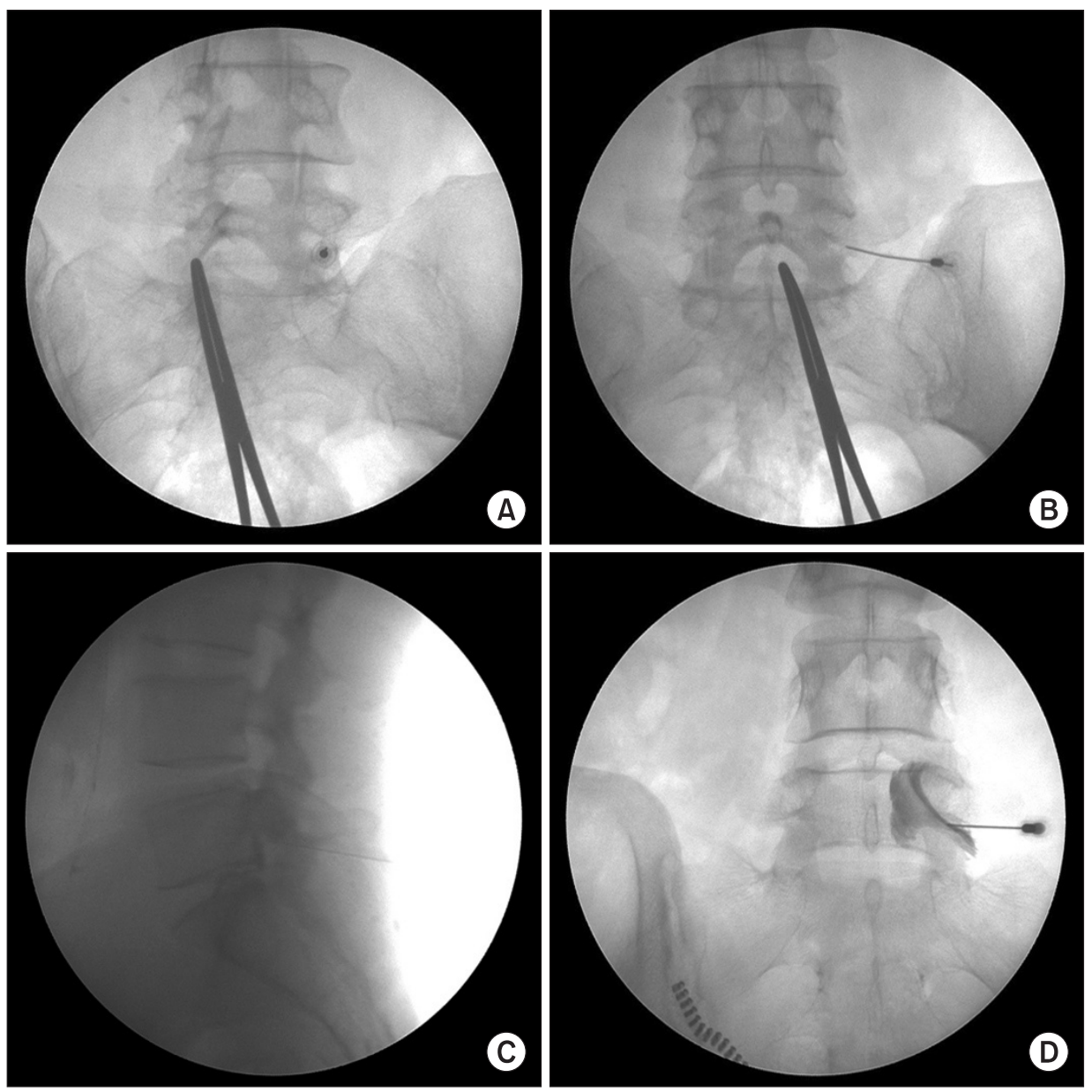

Fig. 4. Subpedicular injection L5S1. (A) In oblique view, needle tip lies directly inferior to the pedicle and inferolateral to the pars interarticularis. (B) The AP view showing the proper location of the needle at the base of pedicle. (C) The lateral radiography should also be used while the needle is advanced until the needle tip is at the posterior and superior aspect of intervertebral neural foramen. (D) A small amount of contrast is used to confirm epidural spread.

\section{RESULTS}

\section{The patient flow}

A total of 81 patients were enrolled in this study. Out of this number, seven patients dropped out according to the exclusion criteria. Simple randomization was used to assign 74 subjects to one of two study groups: As a result, the subpedicular approach and the retrodiscal approach groups included 42 patients and 32 patients, respectively. However, five of the subpedicular group subjects and eight of the retrodiscal group subjects were excluded halfway through the study. Two subjects in the subpedicular group and four in the retrodiscal group were dropped from the study because they were treated with drugs that could influence the effectiveness of the epidural block. In addition, three patients in the subpedicular group and four patients in the retrodiscal group were excluded halfway through the study because it became difficult for them to visit the hospital.
Degrees of temporary diagnostic pain relief before and after injection and possible complications during injection

Two weeks after treatment, temporary diagnostic pain relief (as assessed on the VAS scale) significantly reduced in both groups, but there was no significant difference between the two groups in the initial pain intensity, postinjection pain intensity, and post-injection changes in pain (Table 3). The cases in which the contrast agent spread to the upper and lower nerve roots beyond the L5 nerve root were more frequent in the retrodiscal group, which was only ten patients in the subpedicular group compared to sixteen in the retrodiscal group. One case in the retrodiscal group and ten cases in the subpedicular group presented symptoms of nerve root irritation caused by the injection needle during neurosurgery treatment. Both groups showed no complication of nerve root injury, and the inadvertent injection into the intervertebral disc was detected in two patients, one case 
Table 3. Visual Analogue Scale before and after Procedure

\begin{tabular}{lrrc}
\hline & $\mathbf{R D}(\mathbf{n}=\mathbf{2 4})$ & $\mathrm{SP}(\mathbf{n}=\mathbf{3 7})$ & $\mathbf{p}$-value \\
\hline Initial VAS & $7.3 \pm 1.2$ & $7.7 \pm 1.1$ & 0.233 \\
Final VAS & $3.1 \pm 1.6$ & $3.2 \pm 2.6$ & 0.812 \\
Change of VAS & $-4.2 \pm 1.6$ & $-4.4 \pm 2.4$ & 0.657 \\
\hline
\end{tabular}

Values are mean \pm standard deviation

VAS: Visual analogue scale, RD: Retrodiscal group, SP: Subpedicular group

Table 4. Strengths and Weaknesses during Procedure

\begin{tabular}{lccc}
\hline & $\mathbf{R D}$ & $\mathbf{S P}$ & $\mathbf{p}$-value \\
& $(\mathbf{n}=\mathbf{2 4})$ & $(\mathbf{n = 3 7 )})$ & \\
\hline $\begin{array}{l}\text { Dye spread beyond } \\
\text { L5 root }\end{array}$ & 16 & 10 & 0.002 \\
Nerve root irritation & 1 & 10 & 0.038 \\
Nerve root damage & 0 & 0 & 1.000 \\
Intradiscal injection & 1 & 1 & 0.495 \\
\hline Intravascular injection & 0 & 3 & 0.272 \\
\hline
\end{tabular}

RD: Retrodiscal group, SP: Subpedicular group

in each group. The intravascular injection of contrast medium was reported in three cases, all of them in the subpedicular group (Table 4).

\section{DISCUSSION}

The subpedicular approach has been mainly used for transforaminal lumbar epidural blocks. The method of injecting a drug through the safe triangle into the anterior epidural space, as described by Bogduk, ${ }^{4}$ allows a steroid or anesthetic preparation to be injected more selectively and effectively around the nerve roots. However, Lee et al. ${ }^{9}$ asserted that, when subpedicular technique, the drug often flowed into the distal portion along the spinal nerve. Furthermore, in cases of severe foraminal stenosis, it was often difficult to insert the injection needle. Lew et al. ${ }^{10}$ reported that in most cases of lumbar radiculopathy by either herniated disc or foraminal stenosis, the subpedicular approach was not successful for injecting a drug into the epidural space of the intervertebral disc proximal to either the upper part (i.e. the site of the primary lesion) or the proximal part of spinal ganglion. In these cases, it is likely that the retrodiscal approach can be used as an alternative to the subpedicular approach.

Complications of transforaminal epidural blocks include direct nerve root damage by the needle, nerve infarction, and hematoma due to vascular injury. In addition, other possible complications that may result from transforaminal lumbar epidural steroid or anesthetic injections have been reported to account for about $9.6 \%$ of all cases. ${ }^{11}$ Upon transforaminal injection, cases of intravascular drug injection were reported at $9-26 \% .{ }^{12-15}$ Derby et al. ${ }^{16}$ reported that 0.56 percent of them were cases of intra-arterial drug injection which may trigger severe disorders. To prevent the occurrence of such complications, we should be familiar with the anatomical locations of nerves and blood vessels in the intervertebral foramen.

The Adamkiewicz artery is a psoas artery to which the greatest attention should be paid upon lumbar epidural injections. In $80 \%$ of healthy people, when penetrating the spinal canal, the artery enters the intervertebral foramen between left $\mathrm{T} 9$ and $\mathrm{L} 1$.

However, care should be taken because there are some cases (about 20\%) where it enters the intervertebral foramen between L2 and L4. The main trunk of the Adamkiewicz artery enters the medial spinal canal through either the mid or the rostral portion of the foramen. There it passes through the proximal portion of the dorsal root ganglion and the ventral root complex, as previously reported. ${ }^{17}$ Therefore, the subpedicular approach is likely to damage blood vessels such as the Adamkiewicz artery or to trigger complications, such as spinal cord infarction resulting from the intravascular injection of particulate steroids, because the injection needle is placed in the anterior superolateral aspect of the intervertebral foramen. In contrast, the retrodiscal approach is likely to show less risk of such complications because the needle is placed in the appropriate position under the mid portion of the intervertebral foramen. With regard to the inadvertent intravascular injection of contrast medium, the present study found three cases in the subpedicular group and no case in the retrodiscal group.

Radicular pain may sometimes become more severe following a transforaminal epidural block. On average, the incidence of this condition was reported as $4 \%{ }^{18,19}$ Such a complication was expected to occur as the needle was advanced or the drug was injected into the narrowed spinal canal, due to a herniated disc, spinal stenosis or epidural fibrosis. ${ }^{11}$ In this study, its incidence was ten 
cases $(27 \%)$ in the subpedicular group and one case $(0.04 \%)$ in the retrodiscal group, showing a statistically significant difference between the two groups $(\mathrm{p}<0.05)$. The subpedicular injection approach gave a feeling of discomfort as a the drug stimulated the nerve roots when injected in a large dose through the stenosis region. However, the retrodiscal injection approach is less likely to cause irritation because the drug is injected at the posterior aspect of the intervertebral disc, or at the first portion of the spinal canal. Thus, it enters the target nerve root slowly, instead of going right into the stenosis region.

The retrodiscal approach has a high risk of injecting a contrast agent into the intervertebral disc because the drug is injected in the proximity of the intervertebral disc. For the prevention of this risk, a Tuohy needle with a slightly curved tip is considered to be safer. In addition, the position of the needle should be carefully monitored with a fluoroscopic device. In the event of drug injection into the intervertebral disc, the use of an antibiotic for prevention of complications, such as discitis, should be considered. ${ }^{20}$ In this study, the injection of contrast medium into the intervertebral disc was found in two cases, one in each studied group. Both patients did not show discitis or any other complications resulting from the injection event.

After treatment, the degree of temporary diagnostic pain relief (VAS) was significantly reduced in both groups, and there was no significant difference between the two groups in the initial pain intensity, post-injection pain intensity and post-injection changes in pain. The L5-
S1 retrodiscal approach is designed to inject a drug into the anterior epidural space of the L5 and the proximal portion of the S1 nerve root, and the administered contrast medium reaches all regions, including the proximal portion of the S1 nerve root, the distal portion of the L5 nerve root, and the anterior epidural space. In a study that observed the patterns of contrast spread upon application of the retrodiscal approach, it was found that when the contrast medium was injected into the L5-S1 intervertebral disc, it reached the L5 nerve root in $60.6 \%$, the $\mathrm{L} 5$ and $\mathrm{S} 1$ nerve roots in $30.3 \%$, and the S1 nerve root in $9.1 \%$ ( 3 cases) of the cases. Consequently, there was a statistically significant difference between the retrodiscal approach and the subpedicular approach in the number of cases where a drug was injected into the target nerve root and the proximal portion of the S1 nerve root. ${ }^{21}$ This showed that the administered drug was effective in the spinal ganglion, as well as the L5 nerve root. Therefore the retrodiscal approach was not thought to show a difference in the degree of temporary diagnostic pain relief, compared to the subpedicular injections (Fig. 5). The two approaches were compared in terms of the degree of temporary diagnostic pain relief for two weeks. Future studies are needed to compare treatment effects for at least three months. This study compared the patterns of contrast injection and the possible complications that may occur during injection. However, we did not compare the long-term treatment effects or functional effects of the retrodiscal and subpedicular approaches, and future studies are needed to address these effects.
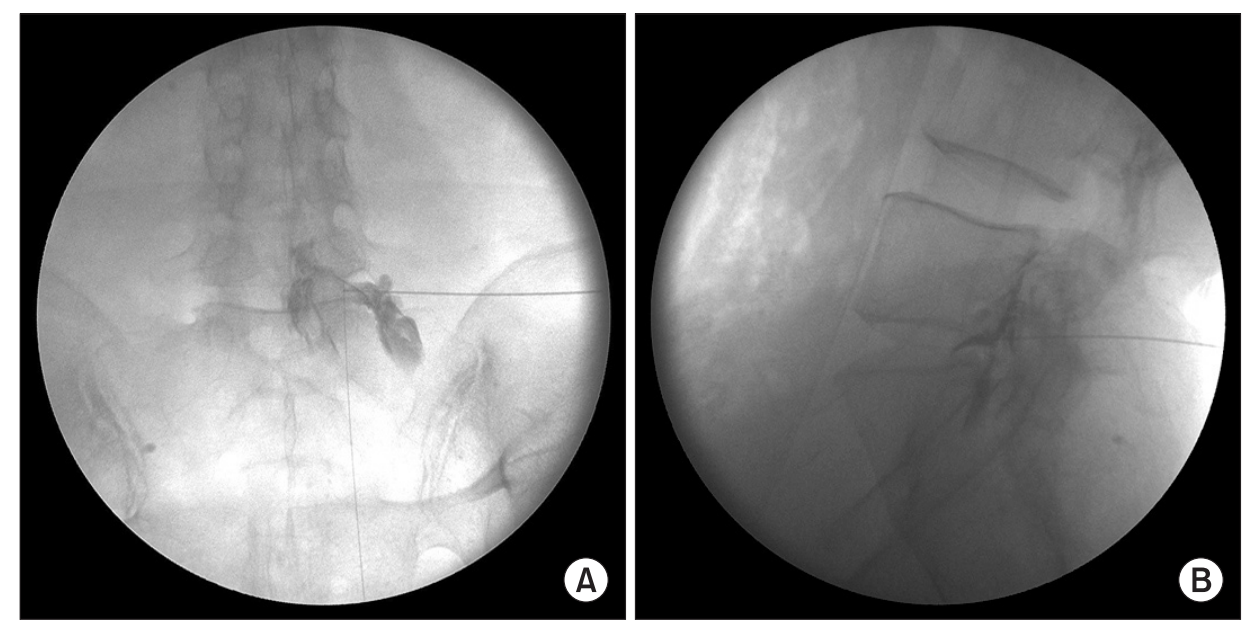

Fig. 5. Posteroanterior spot radiography shows contrast material has spread to L5-S1 disc through the epidural space. The AP view (A) and the lateral view (B). 


\section{CONCLUSION}

When using the L5-sacrum retrodiscal injection approach, epidural blocks can reduce the risk of nerve root irritation during treatment. However, with regard to short-term treatment effects, this procedure shows no difference compared to the subpedicular approach. The retrodiscal injection can be an alternative to the subpedicular approach in specific cases, such as an intervertebral disc sequestrum is incarcerated into the intervertebral foramen or a severe foraminal stenosis.

\section{ACKNOWLEDGEMENT}

This work was supported by Inje University research grant, 2009.

\section{REFERENCES}

1. Botwin KP, Gruber RD, Bouchlas CG, Torres-Ramos FM, Sanelli JT, Freeman ED, Slaten WK, Rao S. Fluoroscopically guided lumbar transforaminal epidural steroid injections in degenerative lumbar stenosis: an outcome study. Am J Phys Med Rehabil 2002; 81: 898-905

2. Slipman CW, Chow DW. Therapeutic spinal corticosteroid injections for the management of radiculopathies. Phys Med Rehabil Clin N Am 2002; 13: 697 711

3. Vad VB, Bhat AL, Lutz GE, Cammisa F. Transforaminal epidural steroid injections in lumbosacral radiculopathy: a prospective randomized study. Spine 2002; 27: 11-16

4. Bogduk N. Clinical anatomy of the lumbar spine and sacrum. 4th ed. New York: Churchill Livingstone, 2005, 123-124

5. Houten JK, Errico TJ. Paraplegia after lumbosacral nerve root block: report of three cases. Spine J 2002; 2 : 70-75

6. Somayaji HS, Saifuddin A, Casey AT, Briggs TW. Spinal cord infarction following therapeutic computed tomography-guided left L2 nerve root injection. Spine 2005; 30: 106-108

7. Stretanski MF, Chopko B. Unintentional vascular uptake in fluoroscopically guided, contrast confirmed spinal injections: a 1-yr clinical experience and discussion of findings. Am J Phys Med Rehabil 2005; 84: 30-35

8. Jasper JF. Lumbar retrodiscal transforaminal injection. Pain Physician 2007; 10: 501-510

9. Lee IS, Kim SH, Lee JW, Hong SH, Choi JY, Kang HS, Song JW, Kwon AK. Comparison of the temporary diagnostic relief of transforaminal epidural steroid injection approaches: conventional versus posterolateral technique. AJNR Am J Neuroradiol 2007; 28: 204-208

10. Lew HL, Coelho P, Chou LH. Preganglionic approach to transforaminal epidural steroid injections. Am J Phys Med Rehabil 2004; 83: 378

11. Botwin KP, Gruber RD, Bouchlas CG, Torres-Ramos FM, Freeman TL, Slaten WK. Complications of fluoroscopically guided transforaminal lumbar epidural injections. Arch Phys Med Rehabil 2000; 81: 1045-1050

12. Sullivan WJ, Willick SE, Chira-Adisai W, Zuhosky J, Tyburski M, Dreyfuss P, Prather H, Press JM. Incidence of intravascular uptake in lumbar spinal injection procedures. Spine 2000; 25: 481-486

13. Furman MB, O'Brien EM, Zgleszewski TM. Incidence of intravascular penetrati on in transforaminal lumbosacral epidural steroid injections. Spine 2000; 25: $2628-2632$

14. Furman MB, Giovanniello MT, O'Brien EM. Incidence of intravascular penet ration in transforaminal cervical epidural steroid injections. Spine 2003; 28 : 21-25

15. Smuck M, Fuller BJ, Yoder B, Huerta J. Incidence of simultaneous epidural and vascular injection during lumbosacral transforaminal epidural injections. Spine J 2007; 7: 79-82

16. Derby R, Lee SH, Kim BJ, Chen Y, Seo KS. Complications following cervical epidural steroid injections by expert interventionalists in 2003. Pain Physician 2004; $7: 445-449$

17. Alleyne CH Jr, Cawley CM, Shengelaia GG, Barrow DL. Microsurgical anatomy of the artery of Adamkiewicz and its segmental artery. J Neurosurg 1998; 89: 791795

18. Burn JM, Langdon L. Lumbar epidural injection for the treatment of chronic sciatica. Rheum Phys Med 1970; 10: 368-374

19. Snoek W, Weber H, Jorgensen B. Double blind evalu- 
ation of extradural methyl prednisolone for herniated lumbar discs. Acta Orthop Scand 1977; 48: 635-641

20. Cohen SP, Maine DN, Shockey SM, Kudchadkar S, Griffith S. Inadvertent disk injection during transforaminal epidural steroid injection: steps for prevention and management. Pain Med 2008; 9: 688-694
21. Kim C, Choi HE, Kang SH, Lim MH, Editors. The contrast spreading pattern in the retrodiscal approach for transforaminal epidural block. The 38th Annual Conference of the Korean Academy of Rehabilitation Medicine; 2010 Oct 21-23; Seoul, Korea. Seoul: Medrang, 2010 